\title{
NECESSARY AND SUFFICIENT CONDITIONS UNDER WHICH CONVERGENCE FOLLOWS FROM SUMMABILITY BY WEIGHTED MEANS
}

\author{
FERENC MÓRICZ and ULRICH STADTMÜLLER
}

(Received 7 February 2001)

\begin{abstract}
We prove necessary and sufficient Tauberian conditions for sequences summable by weighted mean methods. The main results of this paper apply to all weighted mean methods and unify the results known in the literature for particular methods. Among others, the conditions in our theorems are easy consequences of the slowly decreasing condition for real numbers, or slowly oscillating condition for complex numbers. Therefore, practically all classical (one-sided as well as two-sided) Tauberian conditions for weighted mean methods are corollaries of our two main theorems.
\end{abstract}

2000 Mathematics Subject Classification. 40E05, 40C05.

1. Introduction. Let $\left(s_{k}: k=0,1,2, \ldots\right)$ be a sequence of real or complex numbers and $p=\left(p_{k}\right)$ be a sequence of nonnegative numbers such that $p_{0}>0$ and

$$
P_{n}:=\sum_{k=0}^{n} p_{k} \rightarrow \infty \quad \text { as } n \rightarrow \infty .
$$

The weighted mean (formed with this sequence $p$ ) of the sequence $\left(s_{k}\right)$ is defined by

$$
t_{n}:=\frac{1}{P_{n}} \sum_{k=0}^{n} p_{k} s_{k} \quad \text { for } n \in \mathbb{N}_{0} .
$$

The sequence $\left(s_{k}\right)$ is said to be summable by this weighted mean method (shortly summable $(\bar{N}, p))$ if the sequence $\left(t_{n}\right)$ converges to a finite limit $s$.

It is well known that condition (1.1) is necessary and sufficient that every convergent sequence is summable $(\bar{N}, p)$ to the same limit, that is, the weighted mean method in question is regular. We are interested in the converse implication. Under what conditions does convergence follow from summability by the given weighted mean method? There are many results answering this question see for example, [2, 6, 8, 9, 12]. However, our two main results give necessary and sufficient Tauberian conditions for all such methods and thus contain all particular results of this kind.

2. Main results. Let $\left(\rho_{n}\right)$ be a strictly increasing sequence of positive integers such that $\rho_{n} \rightarrow \infty$ as $n \rightarrow \infty$. We say that $\rho$ is an upper allowed sequence with respect to $p$ if

$$
\liminf _{n \rightarrow \infty} \frac{P_{\rho_{n}}}{P_{n}}>1 .
$$


Similarly, we say that $\rho$ is a lower allowed sequence for $p$ if

$$
\liminf _{n \rightarrow \infty} \frac{P_{n}}{P_{\rho_{n}}}>1
$$

Denote by $\Lambda_{u}$ and $\Lambda_{\ell}$ the classes of all upper or lower allowed sequences, respectively. In the case of real sequences $\left(s_{k}\right)$, we will prove the following one-sided Tauberian theorem.

THEOREM 2.1. Let $\left(s_{k}\right)$ be a sequence of real numbers, which is summable $(\bar{N}, p)$ to a finite limit $s$. Then

$$
\lim _{k \rightarrow \infty} s_{k}=s
$$

if and only if

$$
\begin{aligned}
& \sup _{\rho \in \Lambda_{u}} \liminf _{n \rightarrow \infty} \frac{1}{P_{\rho_{n}}-P_{n}} \sum_{k=n+1}^{\rho_{n}} p_{k}\left(s_{k}-s_{n}\right) \geq 0, \\
& \sup _{\rho \in \Lambda_{\ell}} \liminf _{n \rightarrow \infty} \frac{1}{P_{n}-P_{\rho_{n}}} \sum_{k=\rho_{n}+1}^{n} p_{k}\left(s_{n}-s_{k}\right) \geq 0 .
\end{aligned}
$$

A few remarks are appropriate here.

REMARK 2.2. (i) Obviously, it is sufficient to verify conditions (2.4) and (2.5) for some subclasses $\tilde{\Lambda}_{u}$ and $\tilde{\Lambda}_{\ell}$. A natural type of subclasses is given by the following construction. Define

$$
\rho_{n}^{u}(\lambda):=\min \left\{m>n ; \sum_{k=n+1}^{m} \frac{p_{k}}{P_{k}} \geq \lambda-1\right\} \text { for } \lambda>1,
$$

then we have

$$
P_{\rho_{n}^{u}(\lambda)} \geq P_{n}+P_{n} \sum_{k=n+1}^{\rho_{n}^{u}(\lambda)} \frac{p_{k}}{P_{k}} \geq \lambda P_{n}
$$

and we may consider $\tilde{\Lambda}_{u}:=\left\{\left(\rho_{n}^{u}(\lambda)\right)_{n}, \lambda>1\right\}$ instead of $\Lambda_{u}$.

Analogously, we define

$$
\rho_{n}^{\ell}(\lambda):=\max \left\{m<n ; \sum_{k=m+1}^{n} \frac{p_{k}}{P_{k}} \geq \lambda-1\right\} \text { for } \lambda>1,
$$

then we have

$$
P_{n} \geq P_{\rho_{n}^{\ell}(\lambda)}+P_{\rho_{n}^{\ell}(\lambda)} \cdot \sum_{k=\rho_{n}^{\ell}(\lambda)+1}^{n} \frac{p_{k}}{P_{k}} \geq \lambda P_{\rho_{n}^{\ell}(\lambda)}
$$

and we may consider $\tilde{\Lambda}_{\ell}:=\left\{\left(\rho_{n}^{\ell}(\lambda)\right)_{n}, \lambda>1\right\}$ instead of $\Lambda_{\ell}$.

(ii) Following Schmidt [10] (see also [2, pages 124-125]), a sequence of numbers is slowly decreasing with respect to the method $(\bar{N}, p)$ if the following condition is satisfied:

$$
\lim _{\lambda \rightarrow 1+} \liminf _{n \rightarrow \infty} \min _{n<k \leq \rho_{n}^{u}(\lambda)}\left\{s_{k}-s_{n}\right\} \geq 0
$$


Note that for $m=\rho_{n}^{\ell}(\lambda)+1$, we have

$$
\rho_{m}^{u}(\lambda)-m \geq n-\rho_{n}^{\ell}(\lambda) \quad \text { since } \sum_{v=m+1}^{n} \frac{p_{v}}{P_{v}}<\lambda-1
$$

by the maximality of $\rho_{n}^{\ell}(\lambda)$. Hence

$$
\liminf _{n \rightarrow \infty} \min _{\rho_{n}^{\ell}(\lambda)<k \leq n}\left\{s_{n}-s_{k}\right\} \geq \liminf _{k \rightarrow \infty} \min _{k<n \leq \rho_{k}^{u}(\lambda)}\left\{s_{n}-s_{k}\right\}
$$

and from (2.10) it follows that

$$
\lim _{\lambda \rightarrow 1+} \liminf _{n \rightarrow \infty} \min _{\rho_{n}^{\ell}(\lambda)<k \leq n}\left\{s_{n}-s_{k}\right\} \geq 0
$$

So, we have that (2.10) implies (2.13) and both yield trivially our TC (2.4) and (2.5).

(iii) Conditions (2.4) and (2.5) are independent of each other in general. We refer to the example given in [7, pages 56-57], in case of integral summability $(C, 1)$ on $\mathbb{R}_{+}$. However, the discrete counterpart in case of $(C, 1)$-summability of sequences (i.e., $p_{k}=1, k \in \mathbb{N}_{0}$ ) can easily be adapted.

(iv) The symmetric counterparts of conditions (2.4) and (2.5) can be written as follows:

$$
\begin{aligned}
& \inf _{\rho \in \Lambda_{u}} \limsup _{n \rightarrow \infty} \frac{1}{P_{\rho_{n}}-P_{n}} \sum_{k=n+1}^{\rho_{n}} p_{k}\left(s_{k}-s_{n}\right) \leq 0, \\
& \inf _{\rho \in \Lambda_{\ell}} \limsup _{n \rightarrow \infty} \frac{1}{P_{n}-P_{\rho_{n}}} \sum_{k=\rho_{n}+1}^{n} p_{k}\left(s_{n}-s_{k}\right) \leq 0 .
\end{aligned}
$$

Now, Theorem 2.1 remains valid if conditions (2.4) and (2.5) are replaced by the latter two conditions. As a by-product we obtain the following: assume that a real sequence is summable $(\bar{N}, p)$ to a finite limit; if conditions (2.4) and (2.5) are satisfied, then conditions (2.14) are also true, and vice versa.

(v) Analogously to (ii), we may say that a real sequence $\left(s_{k}\right)$ is slowly increasing with respect to the method $(\bar{N}, p)$ if it satisfies the condition

$$
\lim _{\lambda \rightarrow 1+} \limsup _{n \rightarrow \infty} \max _{n<k \leq \rho_{n}^{u}(\lambda)}\left\{s_{k}-s_{n}\right\} \leq 0 .
$$

As before, this condition implies conditions (2.14).

Next, we consider complex sequences $\left(s_{k}\right)$ and will prove the following two-sided Tauberian theorem.

THEOREM 2.3. Let $\left(s_{k}\right)$ be a sequence of complex numbers, which is summable $(\bar{N}, p)$ to a finite limit s. If one of the following two conditions is satisfied:

$$
\inf _{\rho \in \Lambda_{u}} \limsup _{n \rightarrow \infty}\left|\frac{1}{P_{\rho_{n}}-P_{n}} \sum_{k=n+1}^{\rho_{n}} p_{k}\left(s_{k}-s_{n}\right)\right|=0,
$$


or

$$
\inf _{\rho \in \Lambda_{\ell}} \limsup _{n \rightarrow \infty}\left|\frac{1}{P_{n}-P_{\rho_{n}}} \sum_{k=\rho_{n}+1}^{n} p_{k}\left(s_{n}-s_{k}\right)\right|=0,
$$

then (2.3) holds. Conversely, (2.3) implies both (2.16) and (2.17).

REMARK 2.4. A sequence is said to be slowly oscillating with respect to the method $(\bar{N}, p)$ if

$$
\lim _{\lambda \rightarrow 1+} \limsup _{n \rightarrow \infty} \max _{n<k \leq \rho_{n}^{u}(\lambda)}\left|s_{k}-s_{n}\right|=0 .
$$

Condition (2.16) clearly follows from (2.18).

We note that Theorem 2.1 can be extended to sequences whose terms belong to an ordered space over the real numbers. We do not enter into details, but refer to [5] and also [6] as a pattern, given in the case of $(C, 1)$-summability.

3. Proofs. The following lemma plays a basic role in the proofs of our theorems.

LEMMA 3.1. Let $\left(s_{k}\right)$ be a sequence of complex numbers which is summable $(\bar{N}, p)$ to a finite limit $s$.

(i) If $\rho \in \Lambda_{u}$, then

$$
\lim _{n \rightarrow \infty} \frac{1}{P_{\rho_{n}}-P_{n}} \sum_{k=n+1}^{\rho_{n}} p_{k} s_{k}=s .
$$

(ii) If $\rho \in \Lambda_{\ell}$, then

$$
\lim _{n \rightarrow \infty} \frac{1}{P_{n}-P_{\rho_{n}}} \sum_{k=\rho_{n}+1}^{n} p_{k} s_{k}=s .
$$

Proof. (i) By definition,

$$
\begin{aligned}
\frac{1}{P_{\rho_{n}}-P_{n}} \sum_{k=n+1}^{\rho_{n}} p_{k} s_{k} & =\frac{P_{\rho_{n}}}{P_{\rho_{n}}-P_{n}} t_{\rho_{n}}-\frac{P_{n}}{P_{\rho_{n}}-P_{n}} t_{n} \\
& =t_{\rho_{n}}+\frac{P_{n}}{P_{\rho_{n}}-P_{n}}\left(t_{\rho_{n}}-t_{n}\right)
\end{aligned}
$$

By (2.1) we have

$$
\limsup _{n \rightarrow \infty} \frac{P_{n}}{P_{\rho_{n}}-P_{n}}=\left(\liminf _{n \rightarrow \infty} \frac{P_{\rho_{n}}}{P_{n}}-1\right)^{-1}<\infty .
$$

Thus (3.1) follows from (3.3) and the convergence of $\left(t_{n}\right)$ to $s$.

(ii) By definition,

$$
\begin{aligned}
\frac{1}{P_{n}-P_{\rho_{n}}} \sum_{k=\rho_{n}+1}^{n} p_{k} s_{k} & =\frac{P_{n}}{P_{n}-P_{\rho_{n}}} t_{n}-\frac{P_{\rho_{n}}}{P_{n}-P_{\rho_{n}}} t_{\rho_{n}} \\
& =t_{n}+\frac{P_{\rho_{n}}}{P_{n}-P_{\rho_{n}}}\left(t_{n}-t_{\rho_{n}}\right) .
\end{aligned}
$$

By (2.2) we have

$$
\limsup _{n \rightarrow \infty} \frac{P_{\rho_{n}}}{P_{n}-P_{\rho_{n}}}=\left(\liminf _{n \rightarrow \infty} \frac{P_{n}}{P_{\rho_{n}}}-1\right)^{-1}<\infty .
$$

Thus (3.2) follows from (3.5) and the convergence of $\left(t_{n}\right)$. 
Proof of TheOrem 2.1. Necessity. Assume (2.3), whence the convergence of $\left(t_{n}\right)$ to $s$ follows. To verify (2.4), consider an arbitrary sequence $\rho \in \Lambda_{u}$. By (3.1) we have

$$
\lim _{n \rightarrow \infty} \frac{1}{P_{\rho_{n}}-P_{n}} \sum_{k=n+1}^{\rho_{n}} p_{k}\left(s_{k}-s_{n}\right)=\lim _{n \rightarrow \infty} \frac{1}{P_{\rho_{n}}-P_{n}} \sum_{k=n+1}^{\rho_{n}} p_{k} s_{k}-\lim _{n \rightarrow \infty} s_{n}=s-s=0 .
$$

This means that (2.4) is satisfied even with an equality sign. Condition (2.5) can be proved analogously relying on (3.2).

Sufficiency. Assume that (2.4) and (2.5) together with the convergence of $\left(t_{n}\right)$ to $s$ hold. In order to prove the convergence of $\left(s_{n}\right)$ we choose some $\varepsilon>0$. By (2.4), there exists a sequence $\rho \in \Lambda_{u}$ such that

$$
\liminf _{n \rightarrow \infty} \frac{1}{P_{\rho_{n}}-P_{n}} \sum_{k=n+1}^{\rho_{n}} p_{k}\left(s_{k}-s_{n}\right) \geq-\varepsilon
$$

By (3.1), the left-hand side in (3.8) equals (note that the first term has a limit)

$$
\lim _{n \rightarrow \infty} \frac{1}{P_{\rho_{n}}-P_{n}} \sum_{k=n+1}^{\rho_{n}} p_{k} s_{k}-\limsup _{n \rightarrow \infty} s_{n}=s-\limsup _{n \rightarrow \infty} s_{n} .
$$

Combining (3.8) with (3.9) yields

$$
\limsup _{n \rightarrow \infty} s_{n} \leq s+\varepsilon
$$

On the other hand, by (2.5) there exists a sequence $\rho \in \Lambda_{\ell}$ such that

$$
\liminf _{n \rightarrow \infty} \frac{1}{P_{n}-P_{\rho_{n}}} \sum_{k=\rho_{n}+1}^{n} p_{k}\left(s_{n}-s_{k}\right) \geq-\varepsilon
$$

By (3.2) the left-hand side in (3.11) equals

$$
\liminf _{n \rightarrow \infty} s_{n}-\lim _{n \rightarrow \infty} \frac{1}{P_{n}-P_{\rho_{n}}} \sum_{k=\rho_{n}+1}^{n} p_{k} s_{k}=\liminf _{n \rightarrow \infty} s_{n}-s .
$$

Combining (3.11) with (3.12) gives

$$
s-\varepsilon \leq \liminf _{n \rightarrow \infty} s_{n} .
$$

Now, (2.3) follows from (3.10) and (3.13).

Proof OF TheOrem 2.3. Necessity. This is essentially a repetition of the proof of Theorem 2.1. Therefore it is omitted.

Sufficiency. We assume (2.16) together with the convergence of $\left(t_{n}\right)$ to $s$. For any sequence $\rho \in \Lambda_{u}$ we have

$$
\left|s_{n}-s\right| \leq\left|\frac{1}{P_{\rho_{n}}-P_{n}} \sum_{k=n+1}^{\rho_{n}} p_{k}\left(s_{k}-s_{n}\right)\right|+\left|\frac{1}{P_{\rho_{n}}-P_{n}} \sum_{k=n+1}^{\rho_{n}} p_{k} s_{k}-s\right| .
$$


It follows from (3.1) that

$$
\limsup _{n \rightarrow \infty}\left|s_{n}-s\right| \leq \limsup _{n \rightarrow \infty}\left|\frac{1}{P_{\rho_{n}}-P_{n}} \sum_{k=n+1}^{\rho_{n}} p_{k}\left(s_{k}-s_{n}\right)\right| .
$$

Taking (2.16) into account we obtain

$$
\limsup _{n \rightarrow \infty}\left|s_{n}-s\right|=0
$$

which is equivalent to (2.3) to be proved.

Assuming (2.17), we can prove (2.3) in an analogous way.

4. Special cases. (a) If $p_{k}=1$ for all $k$, then the weighted mean method is the socalled Cesàro method of order 1 , the method $(C, 1)$. Here for the sequence $\rho_{n}^{u}(\lambda)$, $\rho_{n}^{\ell}(\lambda)$ we may choose $\lambda n$ with $\lambda>1$ and $<1$, respectively. In this case our result was proved in [6] and [7, Section 4] (see also [11]). In the real case, the classical one-sided Tauberian condition

$$
j\left(s_{j}-s_{j-1}\right) \geq-H
$$

of Landau [4] implies slow decrease with respect to the method $(C, 1)$. Here and in the sequel, we denote by $H$ some positive constant not necessarily the same at different occurrences. To justify this, let $\lambda>1$, and $1 \leq n<k \leq \lambda n=: \rho_{n}(\lambda)$, then we have

$$
\begin{aligned}
s_{k}-s_{n} & =\sum_{j=n+1}^{k}\left(s_{j}-s_{j-1}\right) \geq-H \sum_{j=n+1}^{k} \frac{1}{j} \\
& \geq-H \int_{n}^{k} \frac{d x}{x}=-H \log \frac{k}{n} \geq-H \log \lambda \longrightarrow 0 \quad \text { as } \lambda \rightarrow 1+.
\end{aligned}
$$

In the complex case, the classical Tauberian condition is

$$
j\left|s_{j}-s_{j-1}\right| \leq H
$$

yielding slow oscillation.

(b) The same sequence $\left(\rho_{n}(\lambda)\right)$ and the same Tauberian conditions can be applied to the cases $p_{k}=(k+1)^{\alpha} L(k)$ with some $\alpha>-1$ and some slowly varying function $L(\cdot)$ (see [1] for the definition of slowly varying functions).

(c) If $p_{k}=1 /(k+1)$, then the weighted mean is the so-called harmonic mean (of first order). We may choose $\rho_{n}^{u}(\lambda)=\left[n^{\lambda}\right], \lambda>1$ where [·] means the integral part. Obviously, we have

$$
\frac{P_{\left[n^{\lambda}\right]}}{P_{n}} \geq 1+\frac{\int_{n+2}^{\left[n^{\lambda}\right]+2} d x / x}{1+\int_{1}^{n+1} d x / x} \geq 1+\frac{\log \left(\left[n^{\lambda}\right]+2\right)-\log (n+2)}{1+\log (n+1)}
$$

and this is bigger than $\lambda-1-\varepsilon_{n}$ with $\varepsilon_{n} \rightarrow 0$. However, this will do the job since we may replace $\lambda$ by $\lambda^{\prime}$ being just a little bit smaller. We note that the results in [8] are not applicable, since $P_{\lambda n} / P_{n} \rightarrow 1$ for all $\lambda>1$. So our condition (2.10) is now of the form

$$
\lim _{\lambda \rightarrow 1+} \liminf _{n \rightarrow \infty} \min _{\log n \leq \log k \leq \lambda \log n}\left(s_{k}-s_{n}\right) \geq 0 .
$$


Furthermore, condition (2.16), expressing slow oscillation, takes the form

$$
\lim _{\lambda \rightarrow 1+} \limsup _{n \rightarrow \infty} \max _{\log n \leq \log k \leq \lambda \log n}\left|s_{k}-s_{n}\right|=0 .
$$

These conditions are for example, implied by the following conditions

$$
(j \log j)\left(s_{j}-s_{j-1}\right) \geq-H, \quad(j \log j)\left|s_{j}-s_{j-1}\right| \leq H,
$$

respectively, which follows by similar arguments as in (4.2).

(d) If $p_{k}=1 /\left((k+1) \ell_{k}\right)$ where $\ell_{k}=\sum_{v=0}^{k} 1 /(k+1)$ then the weighted means $\left(t_{n}\right)$ form the harmonic means of second order. A suitable sequence is now $\rho_{n}^{u}(\lambda)=$ $\exp \left((\log (n+1))^{\lambda}\right)$. Then the condition of slow decrease is given as

$$
\lim _{\lambda \rightarrow 1+} \liminf _{n \rightarrow \infty} \min _{\log \log n<\log \log k \leq \lambda \log \log n}\left(s_{k}-s_{n}\right) \geq 0,
$$

which is, for example, implied by the local condition

$$
(j \log j \log \log j)\left(s_{j}-s_{j-1}\right) \geq-H .
$$

(e) If $p_{k}=\exp \left(k^{\alpha}\right)$ with some $\alpha \in(0,1)$, then we have $P_{n} \sim n^{1-\alpha} \exp \left(n^{\alpha}\right) / \alpha$ and a suitable sequence $\rho_{n}(\lambda)$ is given by $\rho_{n}^{u}(\lambda)=n+(\log \lambda) n^{1-\alpha} / \alpha$ and we can easily write down the appropriate Tauberian conditions.

(f) If $p_{k}=e^{k}$ then we have $P_{n} \sim e^{n+1} /(e-1)$ and we may choose $\rho_{n}(\lambda)=n+1$ for $1<\lambda<e /(e-1)$. Hence the Tauberian condition of slow decrease type is given by

$$
\liminf _{n \rightarrow \infty}\left(s_{n+1}-s_{n}\right) \geq 0,
$$

so the sequence has to be almost nondecreasing. However, in this case the weighted mean method is equivalent with convergence as it can be seen directly from the inverse transform.

OPEN PROBLEM. The one-sided Tauberian condition (4.1) is also a Tauberian condition for the Abel method. Similar results hold for more general power series methods $\left(J_{p}\right)$ with regularly varying weights $p_{k}$ (cf. [3]). The question is whether our Tauberian condition (2.4) and (2.5) is also a Tauberian condition for the associated power series $\operatorname{method}\left(J_{p}\right)$.

ACKNOWLEDGEMENT. This research was completed while the first author was visiting the University of Ulm in September 2000; and it was partially supported by the Hungarian National Foundation for Scientific Research under Grant T 029094.

\section{REFERENCES}

[1] N. H. Bingham, C. M. Goldie, and J. L. Teugels, Regular Variation, Encyclopedia of Mathematics and its Applications, vol. 27, Cambridge University Press, Cambridge, 1987. MR 88i:26004. Zbl 617.26001.

[2] G. H. Hardy, Divergent Series, Oxford, 1949. MR 11,25a. Zbl 032.05801.

[3] R. Kiesel and U. Stadtmüller, Tauberian theorems for general power series methods, Math. Proc. Cambridge Philos. Soc. 110 (1991), no. 3, 483-490. MR 92m:40008. Zbl 748.40005. 
[4] E. Landau, Über die Bedeutung einiger neuer Grenzwertsätze der Herren Hardy und Axer, Prace Mat. Fiz. 21 (1910), 97-117.

[5] I. J. Maddox, A Tauberian theorem for ordered spaces, Analysis 9 (1989), no. 3, 297-302. MR 90i:40015. Zbl 677.40003.

[6] F. Móricz, Necessary and sufficient Tauberian conditions, under which convergence follows from summability $(C, 1)$, Bull. London Math. Soc. 26 (1994), no. 3, 288-294. MR 95g:40011. Zbl 812.40004.

[7] F. Móricz and Z. Németh, Tauberian conditions under which convergence of integrals follows from summability $(C, 1)$ over $\mathbb{R}_{+}$, Anal. Math. 26 (2000), no. 1, 53-61. CMP 1773 357. Zbl 0964.40002.

[8] F. Móricz and B. E. Rhoades, Necessary and sufficient Tauberian conditions for certain weighted mean methods of summability, Acta Math. Hungar. 66 (1995), no. 1-2, 105-111. MR 95m:40010. Zbl 833.40004.

[9] A. Peyerimhoff, Lectures on Summability, Lecture Notes in Mathematics, vol. 107, Springer-Verlag, Berlin, 1969. MR 57\#3684. Zbl 182.08401.

[10] R. Schmidt, Über divergente Folgen und lineare Mittelbildungen, Math. Z. 22 (1925), 89152.

[11] U. Stadtmüller, Tauberian theorems for weighted means of double sequences, Anal. Math. 25 (1999), no. 1, 57-68. MR 2000f:40007. Zbl 0929.40004.

[12] K. Zeller and W. Beekmann, Theorie Der Limitierungsverfahren, Zweite, erweiterte und verbesserte Auflage. Ergebnisse der Mathematik und ihrer Grenzgebiete, vol. 15, Springer-Verlag, Berlin, 1970 (German). MR 41\#8863. Zbl 199.11301.

FERENC MÓRICZ: UniVERSITY OF SZEged, ARADI VÉRTANÚK TERE 1, 6720 SZEged, HungaRY E-mail address: moricz@math.u-szeged.hu

UlRICH STADTMÜLLER: UNIVERSITÄT UlM, ABT. MATH. III, D-89069 UlM, GERMANY

E-mail address: stamue@mathematik.uni-u1m.de 


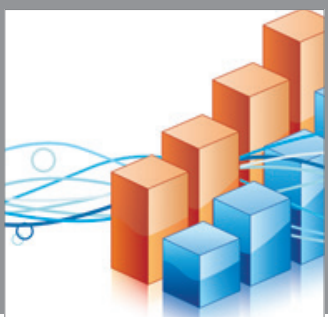

Advances in

Operations Research

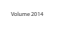

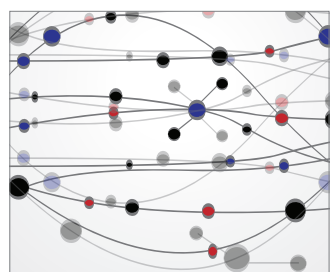

\section{The Scientific} World Journal
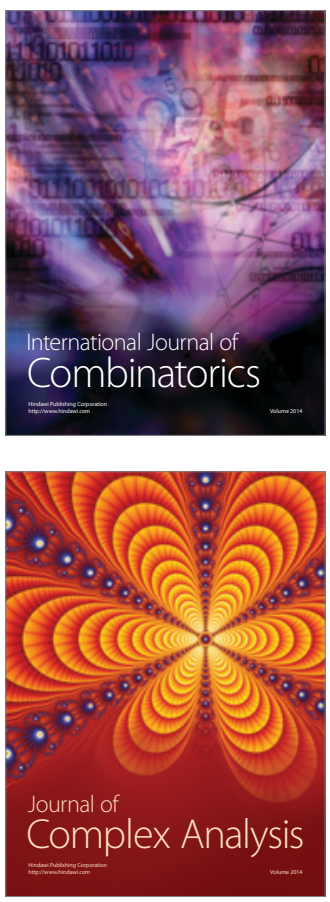

International Journal of

Mathematics and

Mathematical

Sciences
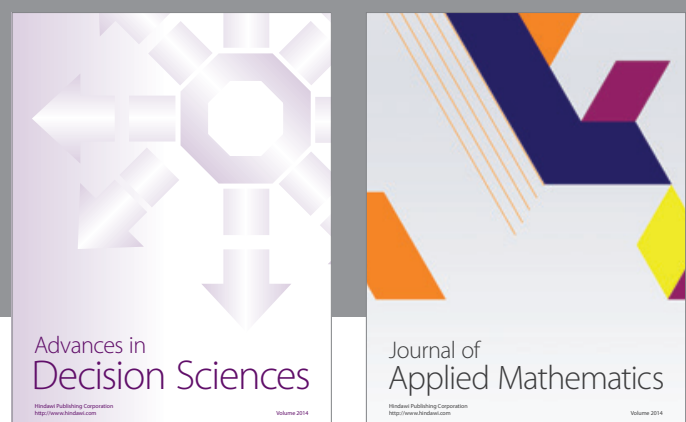

Journal of

Applied Mathematics
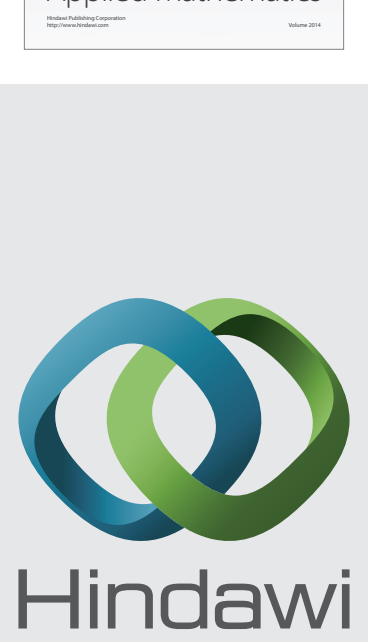

Submit your manuscripts at http://www.hindawi.com
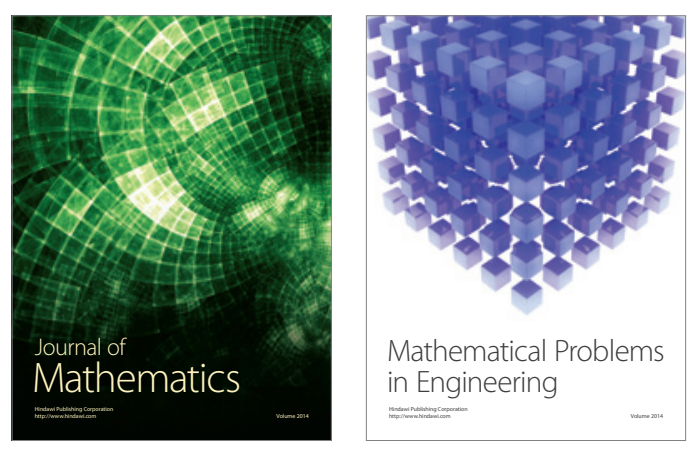

Mathematical Problems in Engineering
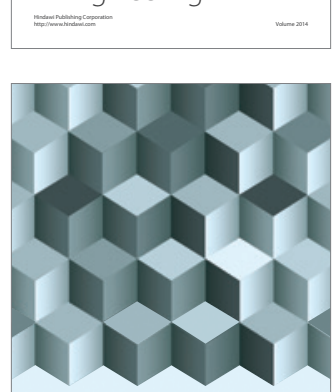

Journal of

Function Spaces
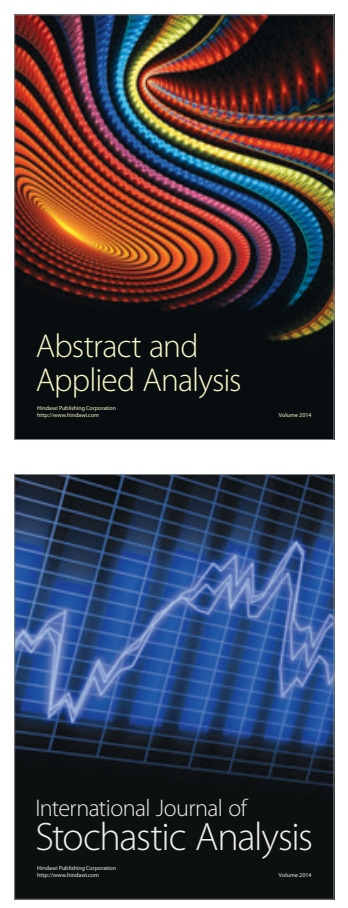

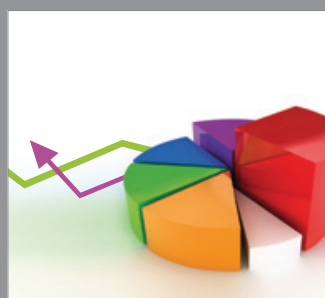

ournal of

Probability and Statistics

Promensencen
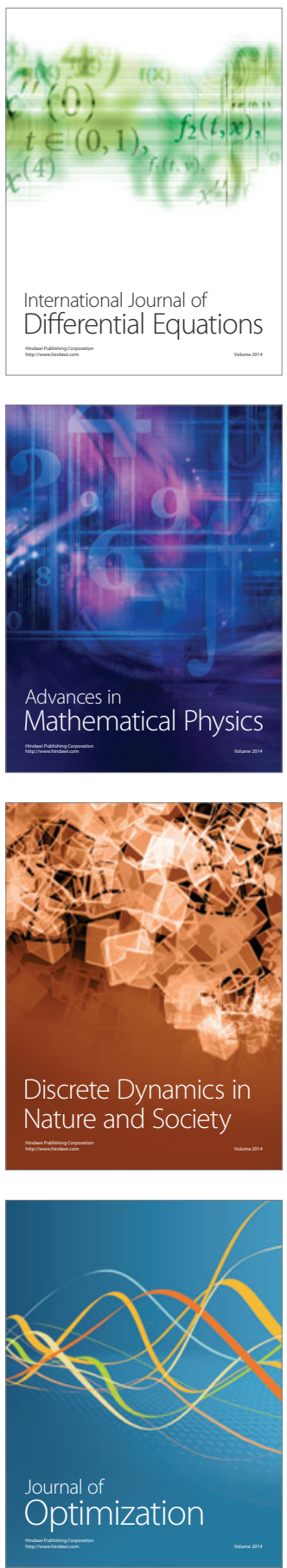